\title{
1. Purity monitoring for ProtoDUNE
}

\section{Richard Diurba for the DUNE Collaboration ${ }^{1, *}$}

$3 \quad$ University of Minnesota

$4 \quad$ E-mail: rdiurba@gmail.com

The next-generation neutrino experiment, DUNE, will utilize a high-intensity neutrino beam produced to measure electron-neutrino appearance and muon-neutrino disappearance with its 40 kilotons (fiducial mass) liquid argon far detector. Liquid argon purity is crucial to use liquid argon time projection chambers (LArTPC) in DUNE's large detectors. A purity monitor is a miniature TPC that measures the lifetime of photoelectrons generated by its UV-illuminated gold photocathode to measure the purity of liquid argon. ProtoDUNEs are full-scale DUNE prototype LArTPCs built at CERN and perform beam tests. The following will discuss the design, implementation, and results of liquid argon purity monitors deployed in ProtoDUNE.

40th International Conference on High Energy physics - ICHEP2020

July 28 - August 6, 2020

Prague, Czech Republic (virtual meeting)

\footnotetext{
${ }^{*}$ Speaker
} 


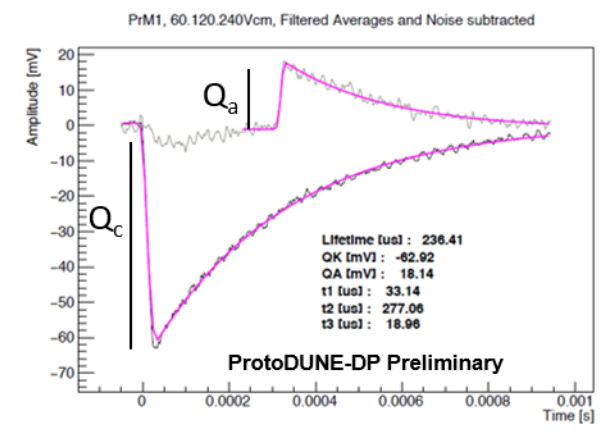

Figure 1: Readout of a purity monitor used for the ProtoDUNE Dual-Phase module.

\section{ProtoDUNE and DUNE Purity Monitors}

The DUNE Far Detector and its prototype program, ProtoDUNE, both use liquid argon time projection chambers (LArTPCs) to detect ionized electrons from charged particles traveling in liquid argon. As these free electrons drift to the readout planes, they can attach to electronegative impurities, such as oxygen and water. The capture of free electron by impurities leads to an attenuation of signals on the LArTPC's readout planes. To compensate, both programs use purity monitors submerged in the liquid argon to quantify the size of this attenuation in order to calibrate out its effects.

These purity monitors have the same design as the purity monitors used by ICARUS T600 [1]. The process of measuring liquid argon attenuation starts with the generation of photoelectrons from a gold photocathode connected to a xenon lamp. These photoelectrons then drift approximately 20 $\mathrm{cm}$ under an electric field from cathode to anode. Due to the photoelectrons inducing a current on the anode and cathode, the total charge on the anode $\left(Q_{a}\right)$ and cathode $\left(-Q_{c}\right)$ can be measured during the photoelectron drift. The ratio of charge on the anode and cathode $\left(-Q_{a} / Q_{c}\right)$ and the total drift time of the photoelectrons $\left(t_{\text {drif } t}\right)$ are used to measure the attenuation by quantifying a drift electron lifetime $(\tau)$. For ProtoDUNE, the single-phase and dual-phase modules use Equation 1 to measure the drift electron lifetime given a sample of signals similar to that of Figure 1.

The DUNE Far Detector technical requirement for the minimum drift electron lifetime for both detector technologies is $3 \mathrm{~ms}$. For reference, using a total drift time for a single-phase module as approximately $2.3 \mathrm{~ms}$, the technical requirement corresponds to at minimum $46 \%$ of charge reaching the readout planes after drifting the $3.6 \mathrm{~m}$ full drift length (Equation 2). For the proposed dual-phase Far Detector module, this value corresponds to only $7 \%$ of charge surviving capture from impurities after traveling the full $12 \mathrm{~m}$ drift length. However, the signal amplification from the charge readout planes allows the dual-phase module to still measure energy deposits despite this attenuation [2].

$$
\tau=\frac{t_{\text {drift } t}}{\ln \left(\frac{Q_{a}}{-Q_{c}}\right)}
$$

$$
\frac{Q\left(t_{\text {full drift }}\right)}{Q(0)}=\exp \left(\frac{-t_{\text {full drift }}}{\tau}\right)
$$




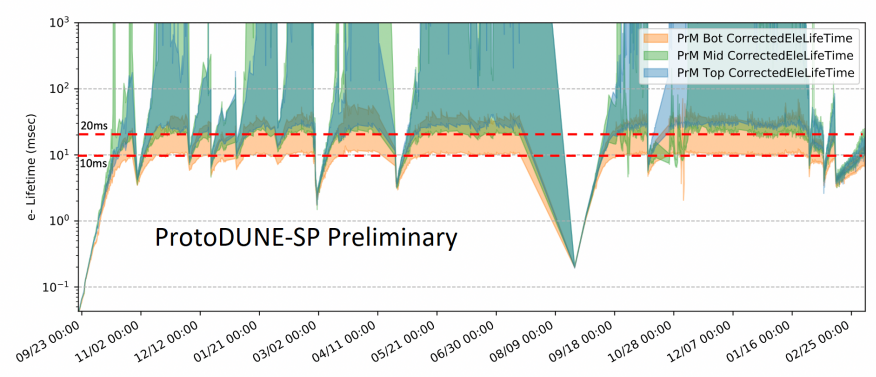

Figure 2: Drift electron lifetime measurements from ProtoDUNE Single-Phase.

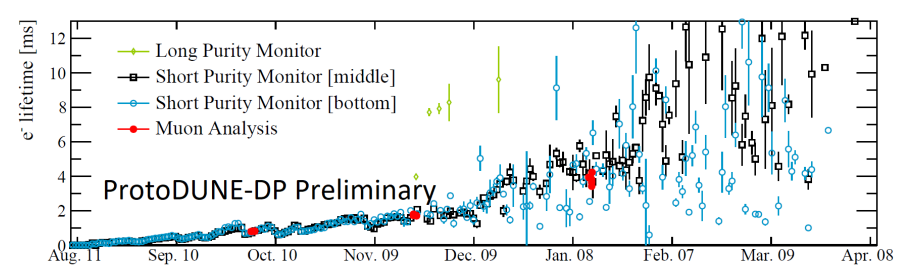

Figure 3: Drift electron lifetime measurements since the beginning of operation of ProtoDUNE Dual-Phase.

\section{Results of ProtoDUNE Purity Monitors}

The ProtoDUNE Single-Phase detector uses three purity monitors placed just outside the TPC. They are vertically separated by around $1 \mathrm{~m}$ from each other. Figure 2 shows that purity monitors measured drift electron lifetimes above $20 \mathrm{~ms}$ for long periods of operation. Measurements of the drift electron lifetime using cosmic muons are in agreement with those measured in Figure 2 [3].

Three purity monitors are used in the dual-phase prototype with one purity monitor placed in the middle of the detector's height and two, including one of much longer drift length, sitting on the detector floor. The electron lifetime measurements from the purity monitors in the dual-phase prototype exceeded the DUNE Far Detector technical requirement in early 2020 and have been corroborated with drift electron lifetime measurements from cosmic muons (Figure 3).

In summary, both ProtoDUNE modules exceed the liquid argon purity guidelines of the DUNE Far Detector and calibrations using the purity monitor readings will be implemented for the data analyses of both detectors.

\section{References}

[1] ICARUS collaboration, S. Amerio et al., Design, construction and tests of the ICARUS T600 detector, Nucl. Instrum. Meth. A527 (2004) 329-410.

[2] DUNE collaboration, B. Abi et al., The DUNE Far Detector Interim Design Report, Volume 3: Dual-Phase Module, 1807.10340.

[3] DUNE collaboration, B. Abi et al., First results on ProtoDUNE-SP liquid argon time projection chamber performance from a beam test at the CERN Neutrino Platform, 2007.06722. 